\title{
REVIEW \\ Virtual feedback for motor and pain rehabilitation after spinal cord injury
}

\author{
M Roosink ${ }^{1,2}$ and C Mercier ${ }^{1,2}$
}

Study design: Interventions using virtual feedback (VF) impact on motor functions and pain and may be relevant for neurorehabilitation after spinal cord injury (SCl) in which motor dysfunctions and (concomitant) pain are frequently observed. Potential mechanisms underlying VF include a modulation of cortical sensorimotor integration, increased therapy engagement and distraction from effort and pain. Still, the optimal parameters for VF and their technical implementation are currently unknown.

Objectives: To provide an overview of interventions that have used VF to improve motor functions or to reduce pain after SCl.

Methods: Literature review.

Results: A total number of 17 studies were identified. VF interventions commonly focused on improving motor functions ( $n=12$ ) or reducing pain $(n=4)$. Only one study assessed both motor functions and pain. Studies generally report beneficial effects. However, the evidence is of low-level quality and many practical as well as theoretical issues remain unclear. Remaining knowledge gaps include: (1) optimal VF system characteristics, (2) the impact of different VF modalities and tasks, (3) dose-response relationships and (4) the identification of patients that are likely to benefit from VF. Future work should start by closing these knowledge gaps using systematic and controlled multi-session interventions and by assessing the underlying mechanisms involved.

Conclusion: These results provide an important incentive to further assess the potential of VF interventions to simultaneously improve motor functions and reduce pain after $\mathrm{SCl}$, which could contribute to better neurorehabilitation outcomes after SCl.

Spinal Cord (2014) 52, 860-866; doi:10.1038/sc.2014.160; published online 30 September 2014

\section{INTRODUCTION}

Technological developments offer new avenues for neurorehabilitation. One such development is virtual feedback (VF). VF uses virtual reality displayed on large screens or on head-mounted displays to provide patients with interactive, multimodal sensory stimuli and biofeedback, and may be applied alone or in combination with physical or cognitive interventions. For example, 'virtual walking', offering an illusion of normal gait, may improve motor functions ${ }^{1,2}$ and may reduce neuropathic pain ${ }^{3-5}$ after spinal cord injury (SCI).

The observation that a single type of VF intervention may impact on motor functions and pain simultaneously ${ }^{6}$ is particularly relevant given the context of intensive neurorehabilitation after SCI during which pain is reported in up to $81 \%$ of patients, ${ }^{7}$ is difficult to treat with conventional therapies, ${ }^{7}$ and interferes with the capacity to perform high-intensity physical activity. ${ }^{8}$ In addition, pain has been shown to directly interfere with sensorimotor functions, such as the capacity to learn locomotor ${ }^{9}$ and upper limb motor ${ }^{10}$ tasks. As such, simultaneous treatment of motor functions and pain would be preferred over conventional treatment, that is suboptimal as evidenced by the high prevalence of chronic pain. ${ }^{7}$ Sequential treatment would be undesirable given the overlap in timing of neurorehabilitation and pain onset ${ }^{7}$ and their negative interaction.

Still, the optimal parameters for VF and their technical implementation are currently unknown. For example, should VF be interactive? What is the optimal duration and frequency of VF sessions? Should VF be combined with other types of therapy, and if yes, how? What patients are likely to benefit from VF? How should we deal with potential adverse effects, for example, pain provocation? ${ }^{11}$

This narrative review paper provides an overview of interventions that have used VF to improve motor functions or to reduce pain after SCI. In addition, this paper addresses potential working mechanisms underlying the therapeutic effects of VF interventions, identifies knowledge gaps and formulates future directions for clinical research into VF interventions targeting motor functions and pain after SCI.

\section{VF INTERVENTIONS FOR PATIENTS WITH SCI: AN OVERVIEW} A literature search was performed to identify original papers, conference proceedings and case studies using VF and/or components of VF (for example, visual feedback or movement observation) on outcome parameters related to lower limb motor functions (including balance) and/or pain after spinal cord injury. Both the SCOPUS and Sciencedirect databases were searched. Search terms included 'spinal cord injury' and 'virtual reality', or 'virtual feedback', 'visual feedback', 'virtual walking', 'visual illusion', 'movement observation', 'action observation', 'passive observation', 'active observation', 'imagery' or 'mental practice'. In addition, the reference lists of selected studies were searched for additional relevant papers. Studies involving a brain computer interface (BCI) to control VF were only included when the $\mathrm{BCI}$ required motor imagery matching the VF task (that is, walking imagery for walking VF). A total number of 17 papers were selected,

${ }^{1}$ Centre Interdisciplinaire de Recherche en Réadaptation et Intégration Sociale (CIRRIS), Québec, Québec, Canada and ${ }^{2}$ Département de réadaptation, Faculté de médecine, Université Laval, Québec, Québec, Canada

Correspondence: Dr M Roosink or Dr C Mercier, Centre Interdisciplinaire de Recherche en Réadaptation et Intégration Sociale (CIRRIS), 525 Boul. Hamel, Québec, Québec, Canada G1M 2S8.

E-mail: meyke.roosink@gmail.com or Catherine.Mercier@rea.ulaval.ca

Received 17 April 2014; revised 5 August 2014; accepted 27 August 2014; published online 30 September 2014 
describing a total number of 13 different VF systems (Table 1). Studies were generally explorative in nature, employing small numbers of patients (1-20 patients per experimental group), and taking place in a clinical setting. VF interventions and assessments were focused on improving motor functions in patients that were pain-free (or in whom pain was not reported; $n=12)^{1,2,12-21}$ or on reducing pain in patients with different levels of motor dysfunction and neuropathic pain $(n=4) .4,5,22,23$ Only one study explicitly assessed the effects of a VF intervention on both motor dysfunctions and neuropathic pain. ${ }^{6}$

\section{VF modalities and tasks}

Improving motor functions. VF interventions focused on walking $(n=4)$, balance $(n=3)$ and leg muscle $(n=2)$ training. Several studies $(n=5)$ involved healthy control subjects to obtain normative data on outcome parameters (for example, electromyography). None of the studies used a VF control intervention. Different VF modalities were used, including combinations of movement execution or motor imagery with interactive VF or movement observation, and simple movement observation (without overt movement execution). VF interfaces included a (TV) screen, LCD monitor or mirror. Only one VF system used a head-mounted display. ${ }^{1,13}$ Interactive VF was mediated by a variety of different sensors (for example, movement, tilt, force, video-capture and electroencephalography). VF tasks included displacement in an environmental scene from a firstperson perspective, controlling virtual limbs from a first-person perspective, controlling a virtual person (avatar) from a third-person perspective, observing one's own movements in a mirror, or the displacement of an object on the screen. Several studies made use of supportive devices, ${ }^{1,2,13,14}$ and one study used neuromuscular electrical stimulation, ${ }^{21}$ to provide active or passive support. A gradation of task difficulty accommodated patients with different levels of baseline functions, and allowed for standardized therapy progression. Therapy could for example be progressed by increasing the required speed or number of targets to be reached, and by decreasing levels of support. Some studies introduced competitive elements, although these were not necessarily associated with additive effects. ${ }^{1,2,13}$ Multi-session interventions applied a total number of 5-48 sessions over a period of 2-16 weeks. ${ }^{12,15,16,19,21}$ The duration ranged from 10 to $60 \mathrm{~min}$ per session.

Reducing neuropathic pain. VF interventions focused on reducing atlevel and below-level neuropathic pain. Only one study used a VF control intervention, ${ }^{5}$ and one other study included both healthy and pain-free SCI patients as control subjects to obtain normative data on outcome parameters (for example, pain thresholds). ${ }^{22}$ Non-interactive video displays and/or a mirror were used. VF tasks included either the observation of video-taped walking legs, synchronized with an upper body mirror-image and (moving) upper limbs to provide an illusion that the patient was walking, or traditional mirror therapy during which movements of the pain-unaffected lower limb had to be observed in a mirror, providing the illusion that the pain-affected limb was moving. Two studies combined VF with transcranial direct current stimulation over the primary motor cortex. ${ }^{5,22}$ Multi-session interventions ${ }^{4,5,22}$ applied a total number of 10-15 sessions over a period of 2-3 weeks. The duration ranged from 10 to $15 \mathrm{~min}$ per session.

Improving motor functions in patients with neuropathic pain. Only one study systematically assessed the effects of a VF intervention on both motor functions and neuropathic pain in patients with SCI having incomplete lesions. ${ }^{6}$ No control intervention was applied. Interactive
VF was displayed on a large screen from a first-person perspective. The movements of the patients were detected by sensors at the feet, and were used to control a pair of virtual legs. By adjusting the sensitivity of the sensors, the actual movements combined with simultaneous observation and imagery of the virtual legs gave patients the illusion that they could use their limbs normally. Several games involving repetitive lower limb movements could be played, such as the juggling of a ball between the two virtual feet. Task progression was based on motor performance. The study applied a total number of 16-20 sessions of $45 \mathrm{~min}$ each over a period of 4 weeks.

\section{Outcome parameters and results}

Motor functions. A variety of outcome parameters have been used to assess motor functions, including VF task performance, activity scores, clinical motor tests, gait speed (10-m walking test), balance (Berg Balance Scale), muscle strength (motor score), mobility (Spinal Cord Independence Measure, Walking Index for Spinal Cord Injury II), locomotion (kinematic gait analysis), global motor effects (Patient Global Impression of Change Scale), electromyography and activity in cortical motor areas.

Single sessions of VF involving walking may lead to increased activity scores, ${ }^{1,13}$ self-confidence and motivation, ${ }^{1,13}$ leg muscle $\mathrm{EMG}^{2}$ and brain activity in motor cortical areas. ${ }^{12,14,15}$ Single and multiple sessions of VF for balance may improve task performance, ${ }^{16-19}$ static and dynamic sitting balance ${ }^{16-19}$ and may increase practice volume and attention span. ${ }^{16}$ Single and multiple sessions of VF for leg muscle functions may increase muscle strength, endurance and ankle joint range of motion, ${ }^{21}$ as well as improve gait patterns, gait speed, muscle strength, balance, ankle dorsiflexion (reducing foot drag) and mobility up to $12-16$ weeks after training. ${ }^{6}$ Although, VF activated motor cortical brain areas, the consistency of the activated regions differed across studies. ${ }^{12,15,20}$ One study showed different brain activation for patients with complete and incomplete lesions. ${ }^{14}$

Neuropathic pain. Outcome parameters included both clinical (for example, pain intensity, unpleasantness, quality, interference, Patient Global Impression of Change Scale) and experimental assessments (for example, pain thresholds, evoked potentials).

In all studies, patients reported overall reduced pain intensities and/ or symptoms after a VF intervention..$^{3-6,23}$ In addition, an increased duration of pain relief, ${ }^{4}$ reduced interference of pain, ${ }^{5}$ increased pain thresholds ${ }^{3}$ and reduced evoked potentials ${ }^{3}$ were reported. Pain reductions were observed up to 12 (see Moseley ${ }^{4}$ and Soler et al. ${ }^{5}$ ) and 16 (see Villiger et al. ${ }^{6}$ ) weeks after therapy termination. In addition, the effects of VF were associated with particular pain qualities (for example, continuous versus paroxysmal pain). ${ }^{5}$ Interestingly, the combination of VF and transcranial direct current stimulation led to significantly more pain reduction than VF or transcranial direct current stimulation alone. ${ }^{5}$

\section{Feasibility and adverse effects}

Feasibility issues such as VF immersion, adherence, drop-out and adverse effects, were generally not systematically assessed. As studies were all clinical lab-based and exploratory, they generally involved the presence of a therapist to ensure a safe therapy session (for example, prevent falls). In those studies that did report adverse effects, these seemed to be occurring in a minority of patients and were considered as relatively mild and comparable to conventional treatment (for example, fatigue, distress and transient pain). ${ }^{1,2,4,6,13,18}$ 


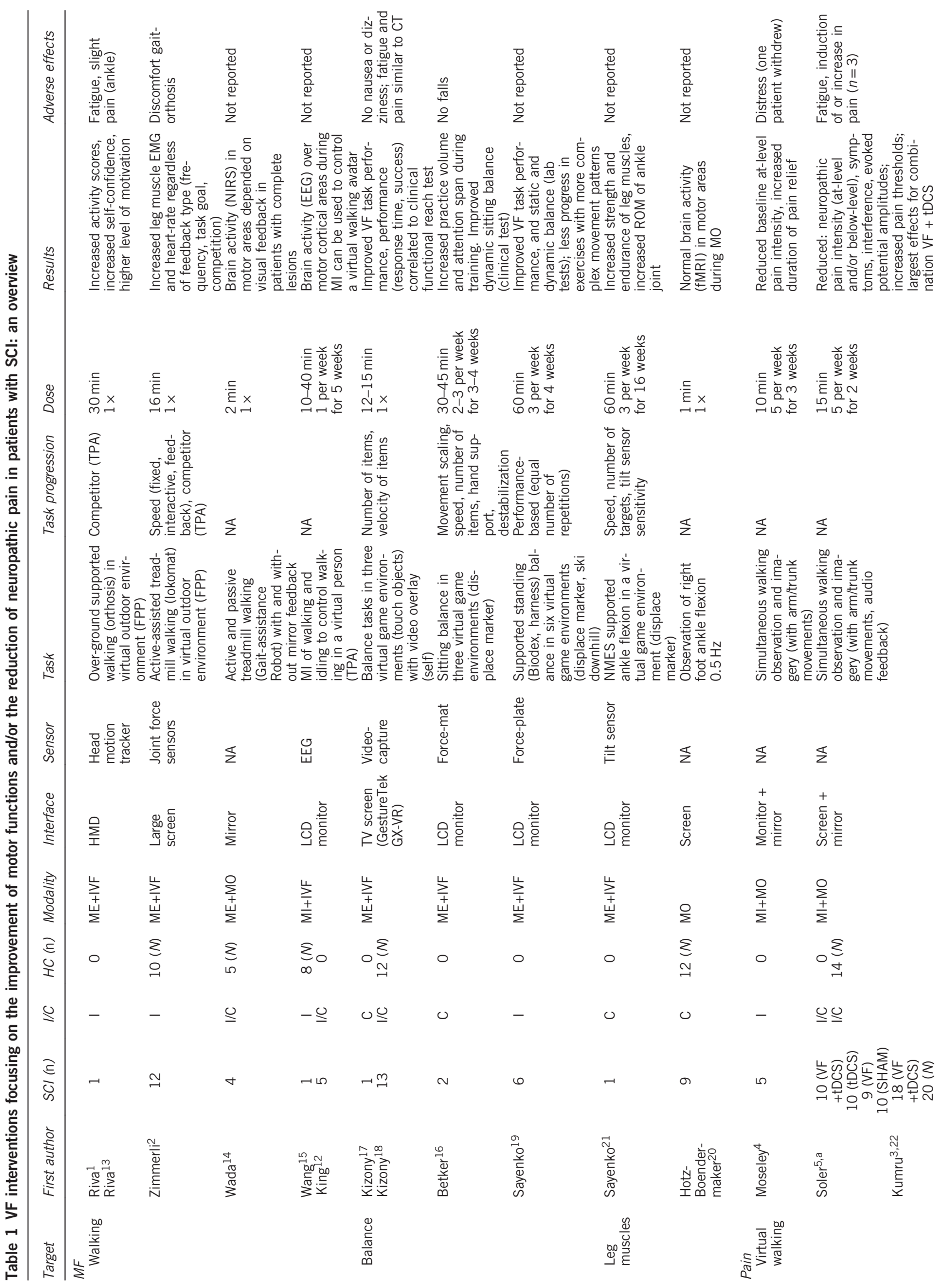


Summary of VF studies in patients with SCI

Several important steps have been made regarding the use of VF interventions to improve motor functions and/or to reduce pain after SCI. Effects were demonstrated even after single session interventions and occurred relatively independent of the precise technical implementation and dose, which were heterogeneously employed. However, when comparing multi-session studies, the dose was usually higher for studies aiming to improve motor functions (up to $45 \mathrm{~min}$ per session, up to $5 \times$ per week, for up to 6 weeks) as compared with studies aiming to reduce pain (up to $15 \mathrm{~min}$, up to $5 \times$ per week, for up to 3 weeks). Importantly, in the single study that assessed both motor functions and pain, higher therapy dose was not associated with an increase in pain. ${ }^{6}$ Providing different gradations in task difficulty seems essential to treat patients with different levels of functions and progression and might be particularly relevant when dealing with concomitant pain (that is, to avoid additional increases in pain or fatigue).

Still, the variability in VF systems, the lack of controlled studies, as well as the relatively small sample sizes, prevent an appropriate evaluation of the efficacy of VF interventions as compared with conventional therapeutic approaches for motor dysfunctions and pain. Moreover, it remains unclear for whom (a particular type of) VF might be most effective. A better understanding of the mechanisms underlying the effects of VF may provide additional guidance for VF research and development and for tailoring VF interventions to individual patients.

\section{POTENTIAL WORKING MECHANISMS UNDERLYING THE EFFECTS OF VF ON MOTOR FUNCTIONS AND PAIN}

Several mechanisms have been proposed to underlie the effects of VF interventions on motor function improvement and pain reduction, including sensorimotor and cognitive-emotional mechanisms. It is assumed that these mechanisms coexist in parallel (rather than operating selectively on motor, sensory or cognitive-emotional functions), and that their relative importance could be related to the design and implementation of VF as well as to SCI characteristics.

\section{VF interventions modulate cortical sensorimotor integration}

In its simplest form VF can be considered as a sensory stimulus which may provide (additional) information about interactions of the body with the environment. For example, the displacement of a virtual object may provide additional information on body positioning and applied force. ${ }^{16,19}$ Moreover, VF interventions may involve movement observation and/or may induce or facilitate motor imagery, which are known to activate a network of brain areas commonly known as the 'mirror system'. ${ }^{24}$ This network of brain areas, including the premotor and primary sensorimotor cortices, is active during the observation, imagery and execution of movements, ${ }^{24}$ and its (repetitive) activation is thought to impact on motor preparation and motor control, ${ }^{25}$ as well as on nociceptive processing ${ }^{26}$ and pain. ${ }^{27}$

After an SCI, structural and functional neuroplastic changes occur both in the spinal cord and brain, and these changes impact on sensorimotor organization and processing (for extensive reviews see Freund et al. ${ }^{28}$ and Kokotilo et al. ${ }^{29}$ ). Interestingly, somatosensory reorganization was found to be more pronounced in SCI patients with below-level neuropathic pain. ${ }^{30}$ As such, the additional sensory information as well as 'mirror system' activation associated with VF interventions may compensate for lacking or altered sensorimotor information after SCI. ${ }^{23}$ In turn, this may contribute to neuroplastic changes that promote a normalization of sensorimotor processing, leading to improvements in motor functions and to reductions in 

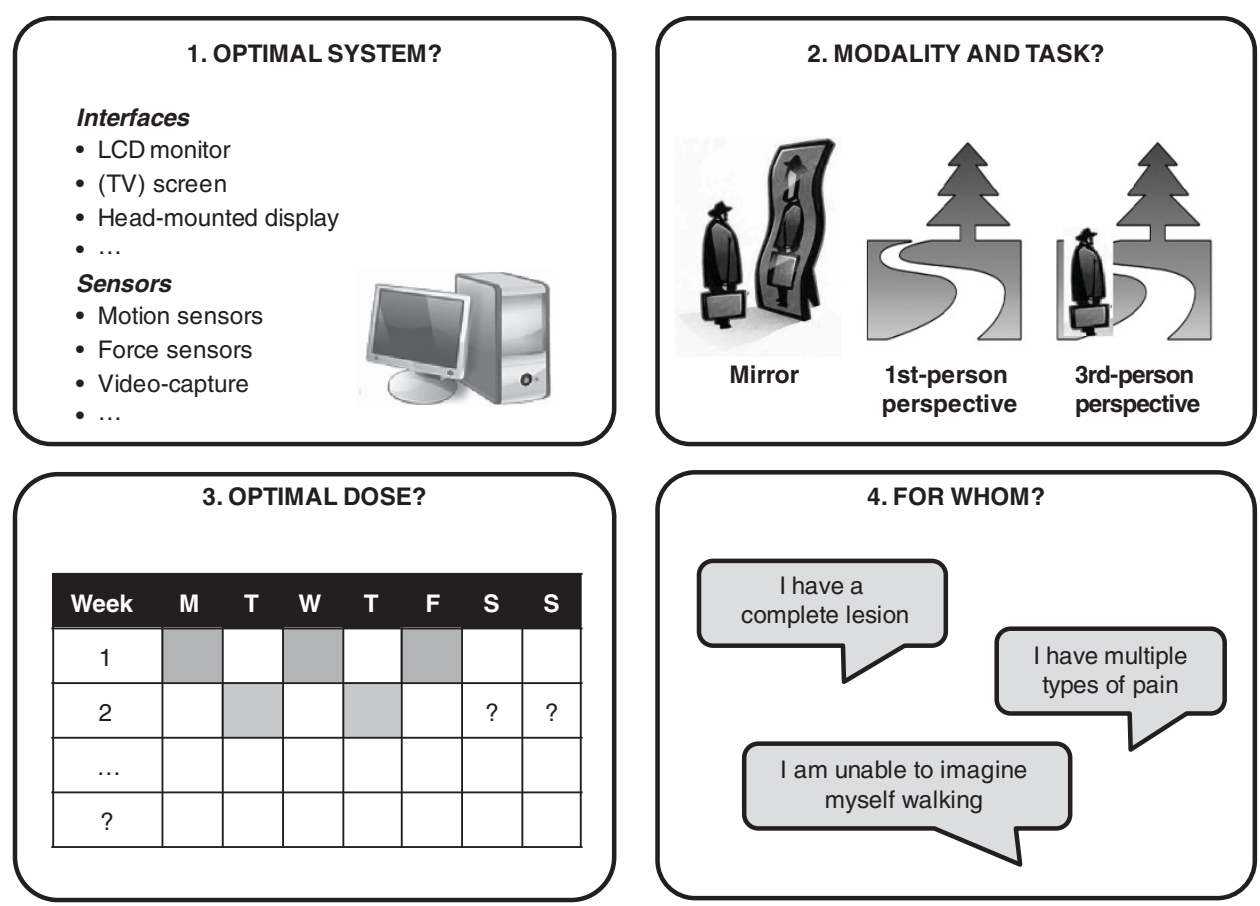

Figure 1 Knowledge gaps relating to the application of VF targeting motor functions and pain in patients with SCI. LCD, liquid crystal display.

pain. ${ }^{25-27}$ The importance of cortical sensorimotor mechanisms underlying the effects of VF seem to be supported by the cumulative rather than independent pain-reducing effects of the combination of VF and transcranial direct current stimulation as observed in patients with neuropathic pain after SCI, ${ }^{5}$ as well as by its simultaneous impact on motor functions and pain. ${ }^{6}$ In addition, motor imagery abilities $^{20,31-34}$ and cortical sensorimotor activity during movement observation, ${ }^{20}$ motor imagery, ${ }^{31,34-36}$ or attempted movements ${ }^{31,34-37}$ have been shown to be intact after SCI, at least for simple movements. Still, these may not be preserved for complex movements. ${ }^{38}$ Moreover, reports of decreased activation levels and delayed timing, ${ }^{35}$ as well as of additional brain regions being activated, ${ }^{31,34}$ suggest that sensorimotor tasks may require more attention after SCI. A better understanding of the cortical sensorimotor mechanisms underlying the effects of VF in patients with SCI is therefore warranted.

VF interventions engage and motivate and may distract from effort and pain

Functional motor recovery has shown to be mainly dependent on therapy intensity and active (goal-oriented) movement repetition. ${ }^{39}$ This requires that attention and motivation are maintained over sustained periods of time in single and multiple training sessions. VF environments and associated games and tasks that transform simple repetitive exercises to goal-oriented movements may contribute to increased and sustained attention levels, intrinsic and extrinsic motivation and an associated sense of reward. In addition, VF immersion may distract patients from potential negative aspects of exercise and from the perception of pain by creating a state of focused concentration on the VF task at hand. This may coincide with a loss of the awareness of oneself, a sense of control over one's activity and an altered sense of time. ${ }^{2}$ Not surprisingly, higher levels of immersion or 'presence' in a virtual environment have been associated with increased distraction and analgesia. ${ }^{40}$ Although distraction is not expected to have long-term effects on the clinical (neuropathic) pain complaints often associated with SCI, it might contribute to the prevention of additional increases in pain and fatigue and as such to increased therapy intensity. Indeed, SCI patients may show increased effort when training in a VF environment, ${ }^{17}$ with interactive $\mathrm{VF}$ leading to particularly high levels of enjoyment, motivation and attention. ${ }^{2,6}$

\section{FUTURE DIRECTIONS: TOWARDS CLOSING KNOWLEDGE GAPS}

On the basis of the overview of studies that aimed to improve motor functions and/or reduce pain in patients with SCI, as well as considering our present understanding of potential mechanisms underlying the effects of VF, the following knowledge gaps have been identified (see Figure 1).

\section{Knowledge gap 1: The optimal VF system}

The acquisition or development of a VF system involves considering different interfaces, sensors and additional feedback modalities. Although it is generally assumed that VF systems inducing higher levels of VF immersion are associated with higher effectiveness, ${ }^{2,6,40}$ this was not explicitly demonstrated by any of the retrieved studies. Rather, it was shown that even a simple mirror may do the trick. As such, more knowledge is needed on the (cost-) effectiveness of different VF systems that aim for similar effects. Still, not only VF immersion could make a particular VF system superior over another. Most VF systems were operated only in the clinical setting and 
required additional safety precautions (for example, to prevent falls or fatigue). As such, the (cost-) effectiveness of a particular VF system is likely to be dependent on the degree to which it can be integrated with, added to, or replace the conventional practice, while remaining safe.

\section{Knowledge gap 2: VF modalities and tasks}

Feedback can be presented from different perspectives, can be a simulation of real-life or involve a non-realistic game environment, and can involve object displacements and/or movement observation. It is likely that particular types of feedback could result in increased immersion and effectiveness, but this remains currently unclear. Interaction is not required per se, since effects on motor functions and pain have also been demonstrated using non-interactive VF. Still, increased levels of VF interaction have been associated with increased muscle activation, ${ }^{2}$ with reduced pain, ${ }^{40}$ and with high levels of enjoyment, motivation and attention. ${ }^{2,6}$ Adding or removing competitive elements or performance feedback could be useful for task progression, but the actual effectiveness of adding these elements has not yet been demonstrated in patients with SCI. ${ }^{1,2,13}$ Theoretically it could be argued that a graded application of VF protocols would be important to avoid additional increases in pain and fatigue, ${ }^{41}$ however, there is currently no evidence that this is indeed the case.

Another element of VF that could impact on levels of VF immersion is the congruency with actual body posture. ${ }^{42}$ Remaining somatosensory afference in incomplete SCI patients might contribute to visual-proprioceptive conflicts when VF is not congruent with body posture. Still, when targeting pain, virtual walking was associated with pain reduction even though applied while patients were sitting. In addition, the possibilities for and safety of applying VF while standing might be limited. As such, a better understanding of the role of body posture and clever solutions for postural support are needed. Finally, for patients with complete lesions, it could be relevant to assess whether VF is associated with attempted versus imaged movements, since attempted movements might increase pain. ${ }^{11}$

\section{Knowledge gap 3: Dose}

Although a variety of VF doses were able to induce effects on motor and pain parameters, none of the selected studies explicitly explored the effectiveness of different doses of VF training. In relation to dose, an important element that remains to be investigated is the use of VF in patients with motor dysfunctions and concomitant pain, which was only explicitly assessed in a single study. ${ }^{6}$ Patients with SCI often have multiple types of pain (for example, musculoskeletal pain, neuropathic pain, visceral pain), ${ }^{7}$ but studies targeting lower limb motor functions have generally performed in patients without (significant) pain, or pain was not assessed or reported. As such, the actual potential of VF protocols to improve motor functions and reduce pain simultaneously remains to be confirmed in future studies.

Knowledge gap 4: Patients likely to benefit from VF interventions Although some studies have hinted towards potentially different effects of VF for patients with complete and incomplete lesions, ${ }^{14}$ and for patients with lower and higher levels of pain, ${ }^{43}$ the relationship between VF effectiveness and patient characteristics remains currently unclear. Although our understanding of mechanisms underlying the effects of VF interventions in patients with SCI is limited due to a lack of mechanistic studies, VF effectiveness is likely to dependent on any factor that could impact on sensorimotor integration, on therapy engagement and motivation and on distraction from effort and pain. As such, it is expected that SCI patients with different age or gender, ${ }^{44}$ somatosensory profiles, ${ }^{45}$ body representations, ${ }^{38,46,47}$ motor imagery abilities $^{48}$ or multiple types of pain ${ }^{5,49}$ may require different types of VF.

\section{Closing the gaps}

Future work should focus on additional multi-session intervention studies including larger sample sizes. Importantly, these should include control interventions and should systematically assess effects on both motor functions and clinical pain, for which the study by Villiger et al. ${ }^{6}$ forms an important starting point. Regarding pain assessment, it will be important to distinguish between temporary task-related fluctuations in pain (that could increase with effort or decrease due to distraction) versus long-term effects on clinical pain (which reflects true clinical outcome). Moreover, as VF could impact differently on different types of pain, a simple numeric rating scale, or even the Basic Pain Data $\mathrm{Set}^{50}$ may not be sensitive enough to demonstrate effects. For this purpose, additional tools assessing different types of pain qualities (for example, neuropathic pain scales) and nociception (for example, quantitative sensory testing) ${ }^{3}$ could be useful. In addition, mechanistic studies are needed to improve our understanding of the role and interaction of cortical sensorimotor integration and cognitive-emotional mechanisms in patients with SCI undergoing VF interventions.

\section{CONCLUSION}

VF may contribute to improve motor functions and reduce pain in patients with SCI, although the evidence so far is of low-level quality. These effects could be mediated by modulations of cortical sensorimotor integration, by increased therapy engagement, and/or by distraction from effort and pain. Considering the high prevalence of concomitant pain and its negative impact on neurorehabilitation outcomes, these results provide an important incentive to further assess the potential of VF interventions to simultaneously improve motor functions and reduce pain after SCI. A better understanding of the underlying mechanisms of VF may guide decisions on VF design and may lead to more effective and tailored VF interventions, which could contribute to better neurorehabilitation outcomes after SCI.

\section{DATA ARCHIVING}

There were no data to deposit.

\section{CONFLICT OF INTEREST}

The authors declare no conflict of interest.

\section{ACKNOWLEDGEMENTS}

MR is supported by a postdoctoral fellowship from the Fonds de recherche Québec-Santé (FRQS) and the SensoriMotor Rehabilitation Research Team (SMRRT) as part of the Regenerative Medicine, and the Nanomedicine Strategic Initiative of the Canadian Institute for Health Research (CIHR). CM is supported by salary grants from CIHR and FRQS, and is a member of the SMRRT team.

1 Riva G. Virtual reality in paraplegia: a VR-enhanced orthopaedic appliance for walking and rehabilitation. Stud Health Technol Inform 1998; 58: 209-218.

2 Zimmerli L, Jacky M, Lünenburger L, Riener R, Bolliger M. Increasing patient engagement during virtual reality-based motor rehabilitation. Am J Phys Med Rehabil 2013; 94: 1737-1746.

3 Kumru H, Soler D, Vidal J, Navarro X, Tormos JM, Pascual-Leone A et al. The effects of transcranial direct current stimulation with visual illusion in neuropathic pain due to spinal cord injury: an evoked potentials and quantitative thermal testing study. Eur $J$ Pain 2013; 17: 55-66. 
4 Moseley GL. Using visual illusion to reduce at-level neuropathic pain in paraplegia. Pain 2007; 130: 294-298.

5 Soler MD, Kumru H, Pelayo R, Vidal J, Tormos JM, Fregni F et al. Effectiveness of transcranial direct current stimulation and visual illusion on neuropathic pain in spinal cord injury. Brain 2010; 133: 2565-2577.

6 Villiger M, Bohli D, Kiper D, Pyk P, Spillmann J, Meilick B et al. Virtual realityaugmented neurorehabilitation improves motor function and reduces neuropathic pain in patients with incomplete spinal cord injury. Neurorehabil Neural Repair 2013; 27 675-683.

7 Siddall PJ, McClelland JM, Rutkowski SB, Cousins MJ. A longitudinal study of the prevalence and characteristics of pain in the first 5 years following spinal cord injury. Pain 2003; 103: 249-257.

8 Tawashy AE, Eng JJ, Lin KH, Tang PF, Hung C. Physical activity is related to lower levels of pain, fatigue and depression in individuals with spinal-cord injury: a correlational study. Spinal Cord 2009; 47: 301-306.

9 Bouffard J, Bouyer LJ, Roy JS, Mercier C. Tonic pain experienced during locomotor training impairs retention despite normal performance during acquisition. J Neurosci 2014; 34: 9190-9195.

10 Lamothe M, Roy J-S, Bouffard J, Gagné M, Bouyer LJ, Mercier C et al. Effect of tonic pain on motor acquisition and retention while learning to reach in a force field. PLoS ONE 2014; 9: e99159.

11 Gustin SM, Wrigley PJ, Gandevia SC, Middleton JW, Henderson LA, Siddall PJ et al. Movement imagery increases pain in people with neuropathic pain following complete thoracic spinal cord injury. Pain 2008; 137: 237-244.

12 King CE, Wang PT, Chui LA, Do AH, Nenadic Z. Operation of a brain-computer interface walking simulator for individuals with spinal cord injury. J Neuroeng Rehabil 2013; 10 77.

13 Riva G. Virtual reality in rehabilitation of spinal cord injuries: a case report. Rehabil Psychol 2000; 45: 81-88.

14 Wada F, Nakanishi Y, Hachisuka K. Effects of gait-related imagery and mirror visual feedback on cortical activation during robot-assisted gait training. ICME International Conference on Complex Medical Engineering. Institute of Electrical and Electronics Engineers (IEEE) Press: Kobe, Japan, 2012.

15 Wang PT, King CE, Chui LA, Do AH, Nenadic Z. Self-paced brain-computer interface control of ambulation in a virtual reality environment. J Neural Eng 2012; 9: 056016.

16 Betker AL, Desai A, Nett C, Kapadia N, Szturm T. Game-based exercises for dynamic short-sitting balance rehabilitation of people with chronic spinal cord and traumatic brain injuries. Phys Ther 2007; 87: 1389-1398.

17 Kizony R, Katz N, Weiss PL. Adapting an immersive virtual reality system for rehabilitation. J Vis Comp Animation 2003; 14: 261-268.

18 Kizony R, Raz L, Katz N, Weingarden H, Weiss PL. Video-capture virtual reality system for patients with paraplegic spinal cord injury. J Rehabil Res Dev 2005; 42: 595-607.

19 Sayenko DG, Alekhina MI, Masani K, Vette AH, Obata H, Popovic MR et al. Positive effect of balance training with visual feedback on standing balance abilities in people with incomplete spinal cord injury. Spinal Cord 2010; 48: 886-893.

20 Hotz-Boendermaker S, Hepp-Reymond MC, Curt A, Kollias SS. Movement observation activates lower limb motor networks in chronic complete paraplegia. Neurorehabi Neural Repair 2011; 25: 469-476.

21 Sayenko DG, Masani K, Milosevic M, Robinson MF, Vette AH, McConville KMV et al. Video game-based neuromuscular electrical stimulation system for calf muscle training: a case study. Med Eng Phys 2011; 33: 249-255.

22 Kumru H, Soler D, Vidal J, Navarro X, Tormos JM, Pascual-Leone A et al. The effects of transcranial direct current stimulation with visual illusion in neuropathic pain due to spinal cord injury: an evoked potentials and quantitative thermal testing study. Eur $J$ Pain 2013; 17: 55-66.

23 Sumitani M, Miyauchi S, McCabe CS, Shibata M, Maeda L, Saitoh Y et al. Mirror visual feedback alleviates deafferentation pain, depending on qualitative aspects of the pain: a preliminary report. Rheumatology (Oxford) 2008; 47: 1038-1043.

24 Jeannerod M. Neural simulation of action: a unifying mechanism for motor cognition. Neuroimage 2001; 14: S103-S109.

25 Mulder T. Motor imagery and action observation: cognitive tools for rehabilitation. J Neural Transm 2007; 114: 1265-1278.

26 Longo MR, lannetti GD, Mancini F, Driver J, Haggard P. Linking pain and the body: neural correlates of visually induced analgesia. J Neurosc 2012; 32: 2601-2607.
27 Moseley GL, Gallace A, Spence C. Bodily illusions in health and disease: physiological and clinical perspectives and the concept of a cortical 'body matrix'. Neurosci Biobehav Rev 2012; 36: 34-46.

28 Freund $\mathrm{P}$, Curt A, Friston K, Thompson A. Tracking changes following spinal cord injury: insights from neuroimaging. Neuroscientist 2013; 19: 116-128.

29 Kokotilo KJ, Eng JJ, Curt A. Reorganization and preservation of motor control of the brain in spinal cord injury: a systematic review. J Neurotrauma 2009; 26: 2113-2126.

30 Wrigley PJ, Press SR, Gustin SM, Macefield VG, Gandevia SC, Cousins MJ et al. Neuropathic pain and primary somatosensory cortex reorganization following spinal cord injury. Pain 2009; 141: 52-59.

31 Alkadhi H, Brugger P, Boendermaker SH, Crelier G, Curt A, Hepp-Reymond MC et al. What disconnection tells about motor imagery: evidence from paraplegic patients. Cerebral Cortex 2005; 15: 131-140.

32 Decety J, Boisson D. Effect of brain and spinal cord injuries on motor imagery. Eur Arch Psych Neurol Sc 1990; 240: 39-43.

33 Fiori F, Sedda A, Ferrè ER, Toraldo A, Querzola M, Pasotti F et al. Motor imagery in spinal cord injury patients: moving makes the difference. J Neuropsychol 2013; 8 : 199-215.

34 Hotz-Boendermaker S, Funk M, Summers P, Brugger P, Hepp-Reymond MC Curt $\mathrm{A}$ et al. Preservation of motor programs in paraplegics as demonstrated by attempted and imagined foot movements. Neuroimage 2008; 39: 383-394.

35 Lacourse MG, Cohen MJ, Lawrence KE, Romero DH. Cortical potentials during imagined movements in individuals with chronic spinal cord injuries. Behav Brain Res 1999; 104: 73-88.

36 Lopez-Larraz E, Antelis JM, Montesano L, Gil-Agudo A, Minguez J. Continuous decoding of motor attempt and motor imagery from EEG activity in spinal cord injury patients. Conf Proc IEEE Eng Med Biol Soc 2012; 2012: 1798-1801.

37 Koenraadt KLM, Duysens J, Rijken H, van Nes IJW, Keijsers NLW. Preserved foot motor cortex in patients with complete spinal cord injury: a functional near-infrared spectroscopic study. Neurorehabil Neural Repair 2014; 28: 179-187.

38 Olsson CJ. Complex motor representations may not be preserved after complete spinal cord injury. Exp Neurol 2012; 236: 46-49.

39 Kwakkel G, Wagenaar RC, Twisk JW, Lankhorst GJ, Koetsier JC. Intensity of leg and arm training after primary middle-cerebral-artery stroke: a randomised trial. Lancet 1999; 354: 191-196.

40 Malloy KM, Milling LS. The effectiveness of virtual reality distraction for pain reduction a systematic review. Clin Psychol Rev 2010; 30: 1011-1018.

41 Bowering KJ, O'Connell NE, Tabor A, Catley MJ, Leake HB, Moseley GL et al. The effects of graded motor imagery and its components on chronic pain: a systematic review and meta-analysis. J Pain 2013; 14: 3-13.

42 Saimpont A, Malouin F, Tousignant B, Jackson PL. The influence of body configuration on motor imagery of walking in younger and older adults. Neuroscience 2012; 222: 49-57.

43 Maani CV, Hoffman HG, Morrow M, Maiers A, Gaylord K, McGhee LL et al. Virtual reality pain control during burn wound debridement of combat-related burn injuries using robot-like arm mounted VR goggles. J Trauma 2011; 71: S125-S130.

44 Triberti S, Repetto C, Riva G. Psychological factors influencing the effectiveness of virtual reality-based analgesia: a systematic review. Cyberpsychol Behav Soc Netw 2014; 17: 335-345.

45 Proske U, Gandevia SC. The proprioceptive senses: their roles in signaling body shape body position and movement, and muscle force. Phys Rev 2012; 92: 1651-1697.

46 Lenggenhager B, Pazzaglia M, Scivoletto G, Molinari M, Aglioti SM. The sense of the body in individuals with spinal cord injury. PLOS ONE 2012; 7: e50757.

47 Fuentes CT, Pazzaglia M, Longo MR, Scivoletto G, Haggard P. Body image distortions following spinal cord injury. J Neurol Neurosurg Psychiatry 2012; 84 201-207.

48 Pernigo S, Moro V, Avesani R, Miatello C, Urgesi C, Aglioti SM et al. Massive somatic deafferentation and motor deefferentation of the lower part of the body impair its visual recognition: a psychophysical study of patients with spinal cord injury. Eur J Neurosc 2012; 36: 3509-3518.

49 Finnerup NB. Pain in patients with spinal cord injury. Pain 2013; 154(Suppl 1): S71-S76.

50 Widerstrom-Noga E, Biering-Sorensen F, Bryce TN, Cardenas DD, Finnerup NB, Jensen MP et al. The International Spinal Cord Injury Pain Basic Data Set (version 2.0). Spinal Cord 2014; 52: 282-286. 